\title{
CARACTERIZAÇÃO SOCIODEMOGRÁFICA E CLÍNICA DE PACIENTES ONCOLÓGICOS
}

\section{CHARACTERIZATION SOCIODEMOGRAPHIC AND CLINICAL OF CANCER PATIENTS}

Monique Pereira Portella, Fernanda Duarte Siqueira, Eliane Raquel Rieth Benetti, Mariléia Stübe, Cibele Thomé da Cruz, Eniva Miladi Fernandes Stumm

\section{RESUMO}

Objetivo: caracterizar pacientes oncológicos internados em um hospital geral com variáveis sociodemográficas e clínicas. Método: estudo transversal, descritivo, quantitativo realizado com 198 pacientes oncológicos hospitalizados nas Clínicas Médica, Cirúrgica e Oncológica, nos meses de dezembro de 2013 a julho de 2014. Os dados foram coletados por meio de Formulário de Caracterização Sociodemográfica e Clínica, analisados com o uso de estatística descritiva. Foram observados os aspectos éticos que regem uma pesquisa com pessoas, projeto de pesquisa aprovado por Comitê de Ética em Pesquisa. Resultados: dentre os participantes, 55,6\% eram do sexo masculino, a maioria com mais de 50 anos de idade, 59,1\% possuíam ensino fundamental incompleto, $63,6 \%$ eram católicos; $27,8 \%$ dos pacientes foram acometidos por câncer do sistema gastrintestinal, $53 \%$ com diagnóstico a menos de um ano. Conclusão: conhecer o perfil dos pacientes assistidos é importante para a equipe multiprofissional, em especial para o enfermeiro, por favorecer e subsidiar 0 planejamento da assistência de enfermagem.

Palavras chave: Pacientes; Oncologia; Hospitalização; Enfermagem.

\section{ABSTRACT}

Objective: to characterize cancer patients admitted to a general hospital with sociodemographic and clinical variables. Methods: cross-sectional, descriptive, quantitative conducted with 198 cancer patients hospitalized in Medical Clinic, Surgery and Oncology, from December 2013 to July 2014. Data were collected through characterization Form Sociodemographic and Clinical analyzed using descriptive statistics. aspects, research project approved by the Research Ethics Committee were observed. Results: of the participants, $55.6 \%$ were male, most with over 50 years of age, $59.1 \%$ had incomplete primary education, $63.6 \%$ were Catholic; $27.8 \%$ of the patients were affected by cancer of the gastrointestinal system, $53 \%$ diagnosed within a year. Conclusion: Knowing the profile of assisted patients is important for the multidisciplinary team, especially for nurses, to favor and support the planning of nursing care.

Descriptors: Patients; Oncology; Hospitalization; Nursing.

Monique Pereira Portella, : Acadêmica de Enfermagem pela Universidade Regional do Estado do Rio Grande do Sul (UNIJUi) que fica na cidade de lijui, Rio Grande do Sul, Brasil.

Fernanda Duarte Siqueira, Acadêmica de Enfermagem pela Universidade Regional do Estado do Rio Grande do Sul (UNIJUi) que fica na cidade de lijui, Rio Grande do Sul, Brasil

Eliane Raquel Rieth Benetti, Mestre em Enfermagem pela Universidade Federal da Santa Maria (UFSM) que fica na cidade de Santa Maria, Rio Grande do Sul, Brasil.

Mariléia Stübe, Enfermeira pela Universidade Regional do Estado do Rio Grande do Sul (UNIJUí) que fica na cidade de liui, Rio Grande do Sul, Brasil.

Cibele Thomé da Cruz, Enfermeira pela Universidade Regional do Estado do Rio Grande do Sul (UNIIUUi) que fica na cidade de ljui, Rio Grande do Sul, Brasil.

Eniva Miladi Fernandes Stumm, Doutora em Ciências (Enfermagem) pela Universidade Federal de São Paulo (UNIFESP) que fica na cidade de São Paulo, São Paulo, Brasil. 
Introdução

Câncer (CA) é o termo utilizado para designar mais de 100 tipos diferentes de doenças que têm em comum o crescimento desordenado de células anormais com potencial invasivo1. Essa doença é responsável por cerca de 13,7\% das mortes no Brasil2 e, conforme dados da Organização Mundial da Saúde (OMS), estimam-se para o ano de 2030, 27 milhões de casos incidentes de câncer, 17 milhões de mortes e 75 milhões de pessoas vivas, anualmente, com câncer3.

Quanto aos tipos de câncer mais frequentes em homens destacam-se os de próstata, pulmão, cólon e reto; nas mulheres os de mama, cólon, reto e pulmão1. Dados da mesma fonte mostram que no Brasil, a estimativa para o ano de 2014 válida também para 2015 é de 576 mil casos novos de câncer1.

Pela incidência e características da doença, a confirmação do diagnóstico de câncer não é fácil tanto para os pacientes quanto para os familiares. Quando o diagnóstico ocorre tardiamente confere maior gravidade para a doença e desse modo implica em sentimentos de medo e insegurança. 0 tratamento é agressivo, causa muitas reações adversas, compartilhada pelo paciente e por sua família com a equipe de saúde. Nesse sentido, o sofrimento do paciente oncológico provocado pela dor e demais efeitos do tratamento requer conhecimento, habilidade técnica e principalmente, empatia carinho e comunicação interpessoal entre pacientes e equipe4.

A exposição das pessoas a fatores potencialmente danosos tem colaborado para a incidência das doenças crônico degenerativas, dentre as quais o câncer. Isso porque, as mudanças atuais nos padrões de vida em relação ao trabalho, nutrição e consumo em geral expõem os indivíduos a fatores ambientais mais agressivos, relacionados a agentes químicos, físicos e biológicos decorrentes do processo de industrialização5. Nesse contexto, considera-se importante que o enfermeiro tenha conhecimento acerca do perfil dos pacientes que assiste com vistas a um planejamento adequado para uma assistência sistematizada e integral, extensiva aos familiares.

No âmbito da enfermagem, especialmente no tratamento oncológico, é desejável a promoção de uma assistência que garanta o cuidado integral, cujas necessidades biopsicossociais e espirituais das pessoas sejam atendidas6. Ademais, em função da multidimensionalidade dos fatores inerentes à patologia e a finitude humana, é essencial que a enfermagem desenvolva o planejamento individualizado da assistência, compreendendo e valorizando a relação destes para enfrentamento da doença, na visão do paciente, com o intuito de uma prática de qualidade 7 .

Frente a estas acepções e dada à importância de se conhecer os diferentes aspectos dos pacientes oncológicos, buscase com o presente estudo responder a seguinte questão: "Quais as características sociodemográficas e clínicas de pacientes oncológicos assistidos em um hospital geral?" Com vistas a responder essa questão, objetiva-se caracterizar um grupo de pacientes oncológicos internados em um hospital geral com variáveis sociodemográficas e clínicas.

Metodologia

Trata-se de um estudo transversal, descritivo, de abordagem quantitativa, com 198 pacientes oncológicos, assistidos em uma instituição de assistência à saúde, no noroeste do estado do Rio Grande do Sul. Foram incluídos pacientes oncológicos hospitalizados, que referiram ou apresentaram sinais de dor, com 18 anos completos ou mais $e$ que aceitaram participar da pesquisa. A coleta ocorreu nas unidades de internação Clínica Médica, Clínica Cirúrgica e Clínica Oncológica, as quais atendiam os respectivos pacientes, nos meses de dezembro de 2013 a julho de 2014. Não participaram do estudo pacientes com dificuldades em compreender e responder às questões contidas no instrumento de coleta de dados.

As entrevistas foram realizadas diariamente no leito do paciente, por acadêmicos de enfermagem, sendo três bolsistas de iniciação cientifica e sete auxiliares de pesquisa voluntarios, previamente instrumentalizados para essa atividade. A coleta de dados foi realizada em prontuário com o objetivo de preencher Formulário de Caracterização Sociodemográfica e Clínica, desenvolvido pelas pesquisadoras, e contemplava as variáveis: nome; idade; sexo; situação conjugal; nível educacional; religião; diagnóstico médico; tempo de diagnóstico; metástase (sim, não, local); recidiva; tratamentos realizados (cirurgia, quimioterapia, radioterapia, quimioterapia + radioterapia); tempo de internação.

Após a coleta os dados foram compilados em uma planilha eletrônica e analisados com o uso de estatística descritiva e com o auxílio do software estatístico Statistical Package for Social Sciences (SPSS), versão 17.0.

Foram observados todos os aspectos éticos que regem uma pesquisa com pessoas, conforme Resolução 4662012 do Ministério da Saúde. O projeto de pesquisa foi aprovado pela comissão de avaliação do Hospital de Caridade de ljuí $(\mathrm{HCl})$ e posteriormente pelo Comitê de Ética em Pesquisa da UNIJUí, sob Parecer Consubstanciado nº 427.613 Ressalta-se que os resultados apresentados integram a pesquisa interinstitucional "Avaliação da dor, estresse e coping em pacientes e familiares no âmbito hospitalar".

\section{RESULTADOS:}

A população estudada compreendeu 198 pacientes com câncer, internados nas unidades de Clínica Médica, Clínica Cirúrgica e Clínica Oncológica assistidos em um hospital do noroeste do Estado do Rio Grande do Sul. Constatouse que, destes, $55,6 \%$ eram do sexo masculino, conforme explicitado na Tabela 1.

Tabela 1 - Características sociodemográficas dos pacientes oncológicos. Rio Grande do Sul, 2016.

\begin{tabular}{|c|c|c|c|}
\hline \multirow[t]{2}{*}{ Características } & \multicolumn{2}{|c|}{ Sexo } & \multirow[b]{2}{*}{ Total n(\%) } \\
\hline & Feminino $n(\%)$ & Masculino n(\%) & \\
\hline \multicolumn{4}{|l|}{ Idade } \\
\hline $18 \mid---30$ anos & $3(1,5)$ & $4(2,0)$ & $7(3,5)$ \\
\hline $31 \mid--40$ anos & $7(3,5)$ & $1(0,5)$ & $8(4,0)$ \\
\hline $41 \mid--50$ anos & $21(10,6)$ & $15(7,6)$ & $36(18,2)$ \\
\hline $51---60$ anos & $21(10,6)$ & $34(17,2)$ & $55(27,8)$ \\
\hline $61 \mid--70$ anos & $20(10,1)$ & $38(19,2)$ & $58(29,3)$ \\
\hline $71 \mid---80$ anos & $14(7,1)$ & $14(7,1)$ & $28(14,1)$ \\
\hline $81 \mid---90$ anos & $2(1,0)$ & $4(2,0)$ & $6(3,0)$ \\
\hline \multicolumn{2}{|c|}{ Média ¿Desvio Padrão (Mínimo;Máximo) } & \multicolumn{2}{|c|}{$58,56 \pm 13,46(18 ; 88)$} \\
\hline \multicolumn{4}{|l|}{ Escolaridade } \\
\hline Sem instrução & $6(3,0)$ & $6(3,0)$ & $12(6,1)$ \\
\hline $\begin{array}{l}\text { Ensino Fundamental } \\
\text { Incompleto }\end{array}$ & $43(21,7)$ & $74(37,4)$ & $117(59,1)$ \\
\hline $\begin{array}{l}\text { Ensino Fundamental } \\
\text { Completo }\end{array}$ & $9(4,5)$ & $9(4,5)$ & $18(9,1)$ \\
\hline $\begin{array}{l}\text { Ensino Médio } \\
\text { Incompleto }\end{array}$ & $2(1,0)$ & $4(2,0)$ & $6(3,0)$ \\
\hline $\begin{array}{l}\text { Ensino Médio } \\
\text { Completo }\end{array}$ & $17(8,6)$ & $14(7,1)$ & $31(15,7)$ \\
\hline $\begin{array}{l}\text { Completo } \\
\text { Graduação }\end{array}$ & $10(5,1)$ & $1(0,5)$ & $11(5,6)$ \\
\hline Pós graduação & $1(0,5)$ & $1(0,5)$ & $2(1,0)$ \\
\hline Sem informação & - & $1(0,5)$ & $1(0,5)$ \\
\hline \multicolumn{4}{|l|}{ Religião } \\
\hline Católico & $53(26,8)$ & $73(36,9)$ & $57(28,8)$ \\
\hline Evangélico & $24(12,1)$ & $33(16,7)$ & $14(7,5)$ \\
\hline Outro & $11(5,5)$ & $4(2,0)$ & $110(55,6)$ \\
\hline Total & $88(44,4)$ & $126(63,6)$ & 198(100) \\
\hline
\end{tabular}


Na Tabela 2 estão descritas as características clínicas dos pacientes oncológico, participantes desse estudo.

Tabela 2 - Dados clínicos dos pacientes oncológicos. Rio Grande do Sul, 2016.

\section{Dados clínicos}

Sexo

Total $\mathrm{n}(\%)$

Femininon(\%) Masculinon(\%)

Diagnóstico

\begin{tabular}{lrrr} 
CA de sistema respiratório & $11(5,6)$ & $18(9,1)$ & $29(14,6)$ \\
CA de sistema tegumentar & $22(11,1)$ & $7(3,5)$ & $29(14,6)$ \\
CA de sistema gastrointestinal & $18(9,1)$ & $37(18,7)$ & $55(27,8)$ \\
CA de sistema reprodutor & $17(8,6)$ & $15(7,6)$ & $32(16,2)$ \\
CA de sistema endócrino & $10(5,1)$ & $7(3,5)$ & $17(8,6)$ \\
CA de sistema neurológico & $1(0,5)$ & $5(2,5)$ & $6(3,0)$ \\
CA de sistema imunológico & - & $5(2,5)$ & $5(2,5)$ \\
CA de sistema linfático & $2(1,0)$ & $5(2,5)$ & $7(3,5)$ \\
CA de sistema urinário & $3(1,5)$ & $5(2,5)$ & $8(4,0)$ \\
CA de sistema esquelético & $1(0,5)$ & - & $1(0,5)$ \\
CA de tecido conjuntivo & $1(0,5)$ & - & $1(0,5)$ \\
Sem especificação de localização & $2(1,0)$ & $6(3,0)$ & $8(4,0)$ \\
& & & \\
\hline
\end{tabular}

Tempo de diagnóstico

\begin{tabular}{crrr}
\hline Menos de 1 ano & $48(24,2)$ & $57(28,8)$ & $105(53,0)$ \\
$1 \mid---5$ anos & $21(10,6)$ & $30(15,2)$ & $51(25,8)$ \\
$5 \mid---10$ anos & $4(2,0)$ & $3(1,5)$ & $7(3,5)$ \\
$10 \mid--15$ anos & $1(0,5)$ & - & $1(0,5)$ \\
Não sabe o diagnóstico & $5(2,5)$ & $6(3,0)$ & $11(5,6)$ \\
Não soube especificar o tempo & $9(4,5)$ & $14(7,1)$ & $23(11,6)$
\end{tabular}

\section{Ocorrência de Metástase}

\begin{tabular}{llll}
\hline Sim & $21(10,6)$ & $32(16,2)$ & $53(26,8)$ \\
Não & $42(21,2)$ & $46(23,2)$ & $88(44,4)$ \\
Sem Informação & $25(12,6)$ & $32(16,2)$ & $57(28,8)$
\end{tabular}

Tratamento Realizado**

\begin{tabular}{lrrr}
\hline Cirurgia & $9(4,6)$ & $8(4,1)$ & $17(8,7)$ \\
Quimioterapia & $10(5,1)$ & $21(10,7)$ & $31(15,8)$ \\
Radioterapia & $2(1,0)$ & $3(1,5)$ & $5(2,6)$ \\
Radioterapia + Quimioterapia & $7(3,6)$ & $11(5,6)$ & $18(9,2)$ \\
Cirurgia, quimioterapia & $9(4,6)$ & $13(6,6)$ & $22(11,2)$ \\
e radioterapia & & & \\
Cirurgia e quimioterapia & $14(7,1)$ & $12(6,1)$ & $26(13,3)$ \\
Cirurgia, quimioterapia, radioterapia & $3(1,5)$ & - & $3(1,5)$
\end{tabular}

e braquiterapia

Cirurgia, quimioterap

e hormonioterapia

Cirurgia e radioterapia

Cirurgia, quimioterapia

e tratamento paliativo no momento

Outro

Nenhum

Sem Informação

$1(0,5)$

Tempo Internação

\begin{tabular}{lrrr}
\hline $0 \mid---10$ dias & $60(39,2)$ & $81(52,9)$ & $141(92,2)$ \\
$11 \mid---20$ dias & $4(2,6)$ & $3(2,0)$ & $7(4,6)$ \\
$21 \mid---30$ dias & - & $4(2,6)$ & $4(2,6)$ \\
$31 \mid---40$ dias & - & $1(0,7)$ & $1(0,7)$ \\
Média $\pm D P$ (Mínimo;Máximo) & & & $4,44 \pm 5,54(0 ; 36)$
\end{tabular}

Média DDP (Mínimo;Máximo) 
percentual maior. Estes resultados são convergentes a estudo que delineou o perfil de 147 pacientes com câncer em tratamento radioterápico em um Centro Regional de Oncologia da Região Sul do Brasil, no qual se identificou que a baixa escolaridade interfere na compreensão quanto ao tratamento, o autocuidado e na relação entre paciente e profissional 10.

Constatou-se que $63,6 \%$ dos pacientes oncológicos participantes da pesquisa professavam a religião católica.

O mesmo foi verificado em pesquisa realizada em duas cidades da Paraíba, em que $71,9 \%$ dos entrevistados eram católicos e 19,3\% evangélicos, independente da religião todos afirmaram ter fé11. Nesse aspecto, estudo realizado em um ambulatório de oncologia de um hospital público de Minas Gerais, com 101 pacientes em quimioterapia, mostrou que o enfrentamento religioso/espiritual traz emoções positivas, que pode minimizar o sofrimento e aumentar a esperança de cura, ou aceitação da doença12. No que se refere a esse aspecto é importante que os profissionais de saúde incentivem tais ações, pois ações direcionadas ao cuidado espiritual são necessárias por oportunizar o cuidado integral aos pacientes oncológicos.

Quanto aos dados clínicos dos pacientes pesquisados, verificou-se que o maior percentual $(27,8 \%)$ era dos que apresentavam CA no sistema gastrointestinal, do sexo masculino (18,7\%), seguido dos com CA no sistema reprodutor $(6,2 \%)$, com maior percentual de mulheres $(8,6 \%)$ e CA nos sistemas respiratório e tegumentar em percentuais idênticos $(14,16 \%)$ porém, mais incidente em mulheres (16,7\%). Autores descreveram em estudo com 221 pessoas com câncer en tratamento em uma unidade oncológica na região sul do Brasil, maior porcentual de mulheres, com baixa escolaridade que possuíam o hábito de tomar chimarrão13. Nesse contexto, salienta-se que tomar chimarrão em temperaturas muito altas, assim como dieta rica em gordura são as principais causas de aumento de câncer do trato gastrointestinal14. 0 hábito do chimarrão são características de indivíduos do sul do país, nesse sentido, neste estudo verificou-se maior percentual de CA no sistema gastrointestinal nessa população13.

Resultados diferentes foram encontrados em estudo em que os dados foram coletados por meio da consulta aos prontuários de pacientes em tratamento quimioterápico ambulatorial no Rio de Janeiro, no qual foi evidenciado que o tipo de CA com maior percentual foi o de mama, seguido do CA de cólon e reto15. Diferente do presente estudo, a pesquisa realizada com prontuários de 180 pacientes com câncer de pulmão em hospital público de referência oncológica do estado do Pará, mostrou que o maior percentual foi de pacientes do sexo masculino nesse tipo de CA16 e estudo de revisão de literatura sobre o perfil de pacientes estomizados, mostrou prevalência do sexo feminino17.

Verificou-se que mais da metade dos pacientes pesquisados (53\%) tinham o diagnóstico de câncer há menos de um ano, seguido dos de 1 a 5 anos incompletos (25,8\%). Outro resultado importante é que $44,4 \%$ deles não apresentaram metástase. Estudo realizado com 164 pacientes com câncer gástrico em Porto Alegre analisou a associação entre metástases linfonodais e diferentes fatores prognósticos em câncer gástrico, mostrou que a presença de metástases esteve associada à profundidade de invasão e tamanho tumoral18. Nesse contexto, o fato de grande parte dos pacientes deste estudo não apresentar metástase pode ter relação com a descoberta recente da doença.

Quanto ao tratamento ministrado, 15,8\% realizaram quimioterapia, seguido de cirurgia e quimioterapia $(13,3 \%)$ e cirurgia, quimioterapia e radioterapia $(11,2 \%)$. A quimioterapia é o tratamento mais temido pelos pacientes pelo fato de desencadear muitas reações. Neste contexto 0 enfermeiro deve estar preparado para planejar, organizar, supervisionar $e$ executar atividades para alivio de sintomas, prevenção e minimização de efeitos colaterais, bem como educar o paciente e familiar sobre o tratamento19.

Estudo realizado no Paraná analisou prontuários de 128 pacientes com câncer de laringe e comparou o tratamento de radioterapia e 0 de cirurgia20. Os autores pontuam que os pacientes submetidos à somente cirurgia oncológica obtiveram uma sobrevida geral de 4,3 anos em média, $12 \%$ deles apresentaram recidiva, enquanto que os pacientes submetidos a apenas radioterapia apresentaram sobrevida geral de 2,8 anos e $15 \%$ deles teve recidiva. Mesmo os pacientes tratados de forma associada obtiveram uma taxa menor de sobrevida ( 3,5 anos) e apresentaram maior taxa de recidiva (14\%) do que os tratados apenas com cirurgia20.

Estudo que caracterizou fatores associados com a recusa da terapia cirúrgica ou de radiação mostra que $0,4 \%$ (de um total de 692.938 pacientes com recomendação de cirurgia) recusaram a cirurgia oncológica e $0,9 \%$ (de um total de 232.189 pacientes com recomendação de radioterapia) recusaram a radiação21. Estes pacientes foram caracterizados como solteiros, não-brancos e asiáticos americanos, com idade avançada e baixa renda anual. Os autores pontuam que a recusa do tratamento está associada com 0 aumento das estimativas de mortalidade por câncer.

Em relação ao tempo de internação, a maioria, 92,2\%, permaneceu internado de 1 a 10 dias. Nesse aspecto, 0 ambiente hospitalar pode trazer medo e insegurança para o paciente, que perde sua privacidade e depende da equipe que vai cuidá-lo, desse modo é importante à presença de um acompanhante com ele, por isso o enfermeiro deve estabelecer e manter comunicação continua, não só com o paciente como também com seu familiar, de modo a acolher ambos 15.

No que refere ao cuidado e a prática profissional do enfermeiro, existem lacunas de conhecimento para 0 atendimento aos pacientes portadores de neoplasias, assim, é necessário investir em educação continuada, o que irà resultar em maior qualidade na assistência a esse perfil de paciente22. Os profissionais de saúde necessitam ampliar o foco de atenção, incluir fatores socioculturais do contexto das pessoas que vivenciam o processo de ter uma doença crônica, como o câncer. Para a enfermagem, isso significa, ultrapassar a dimensão biológica do cuidar, com o intuito de construir, junto com as pessoas acometidas pelo câncer, estratégias de cuidado em consonância com suas concepções e expectativas, para promover qualidade de vida15.

Pontua-se que o conhecimento clínico e sociodemográfico dos pacientes permitem ao enfermeiro a compreensão dos tipos de tratamento, raciocínio clinico frente aos problemas apresentados, bem como torna possivel o cuidado integral9. Nesse sentido, o enfermeiro deve ser capaz de avaliar o paciente de forma integral, respeitar as suas individualidades e assim proporcionar um cuidado humanizado que atende as necessidades de cada indivíduo.

\section{Considerações Finais}

Este estudo possibilitou conhecer o perfil sociodemográfico e clínico dos pacientes oncológicos assistidos em um hospital geral, bem como interagir com eles e seus familiares. Os resultados obtidos, aliados aos posicionamentos dos autores e a interação com pacientes oncológicos e seus respectivos familiares, foi importante para o conhecimento dessa população.

Avalia-se que conhecer o perfil da população assistida é importante para a equipe multiprofissional, em especia para o enfermeiro, por favorecer e subsidiar o planejamento da assistência de enfermagem. Nesse contexto, destaca-se a importância de a equipe de enfermagem conhecer o perfil dos pacientes que assiste, bem como de seus familiares, com vistas a um planejamento de ações para uma assistência integral.

Referências

1. Facina T. Estimativa 2014: incidência de câncer no Brasil. Rev bras cancerol [Internet]. 2014 [acesso em: 10 nov 2015];60(1):63-4. Disponivel em: http://www.inca.gov.br/rbc/n_60/v01/pdf/11-resenha-estimativa-2014-incidencia-decancer-no-brasil.pdf

2. Amador DD, Gomes IP, Coutinho SED, Costa TNA, Collet N. Concepção dos enfermeiros acerca da capacitação no cuidado à criança com câncer. Texto Contexto Enferm [Internet]. 2011 [acesso em: 10 nov 2015];20(1):94-101. Disponível em: http://www.scielo.br/pdf/tce/v20n1/11.pdf

3. Instituto Nacional de Câncer Jose Alencar Gomes da Silva (INCA). Estimativa 2012: incidência de câncer no Brasi [Internet]. Rio de Janeiro: INCA, 2011 [acesso em: 10 nov 2015]. Disponível em: http://portal.saude.sp.gov.br/resources/ ses/perfil/gestor/homepage/estimativas-de-incidencia-de-cancer-2012/estimativas_incidencia_cancer_2012.pdf

4. Rennó CSN, Campos CJG. Interpersonal communication research: valorization of the oncological patient in a high complexity oncology unit. Rev Min Enferm. 2014 jan/mar; 18(1): 106-115. [acesso em: 25 set 2015]. Disponivel em: http:// www.reme.org.br/artigo/detalhes/912

5. Souza RS, Simão DAS, Lima EDRP. Perfil sociodemográfico e clínico de pacientes atendidos em um serviço ambulatorial de quimioterapia paliativa em Belo Horizonte. Rev Min Enferm [Internet]. 2012 [acesso em: 25 jan 2016];16(1):38-47. 
Disponível em: http://www.reme.org.br/artigo/detalhes/498

6. Leite MAC, Nogueira DA, Terra FS. Aspectos sociais e clínicos de pacientes oncológicos de um serviço quimioterápico. Rev Rene [Internet]. 2015 [acesso em: 26 jan 2016]; 16(1):38-45. Disponível em: http://www.revistarene.ufc.br/revista/ index.php/revista/article/viewFile/1833/pdf

7. Sonobe HM, Buetto LS, Zago MMF. Cancer patients' knowledge about their legal rights. Rev Esc Enferm USP [Internet]. 2011 [acesso em: 26 jan 2016]; 45(2):342-8. Disponível em: http://www.scielo.br/pdf/reeusp/v45n2/en_v45n2a05.pdf

8. Sena MF, Costa APS, Ferreira MAF. Características sociodemográficas, clínicas e histopatológica de pacientes com carcinoma epidermóide de lábio: uma análise retrospectiva (1997-2004). Medicina (Ribeirão Preto) [Internet]. 2013 [acesso em: 25 set 2015];46(2):128-34. Disponível em: http://www.revistas.usp.br/rmrp/article/view/62414

9. Silva MM, Silva JA, Esteves LO, Mesquita MGR, Stipp MAC Duarte SCM. Perfil sociodemográfico e clínico de pessoas em tratamento quimioterápico: subsídios para o gerenciamento em enfermagem. Rev Eletr Enf [Internet]. 2013 [acesso em: 26 jan 2016];15(3):704-12. Disponível em: https://www.fen.ufg.br/revista/v15/n3/pdf/v15n3a12.pdf

10. Zillmer JGV, Lima LM, Feijó AM, Schwartz E, Hisse CN, Viegas AC et al. Caracterização dos clientes em tratamento radioterápico em um serviço no sul do brasil. Rev Enferm UFSM [Internet]. 2013 [acesso em: 25 set 2015];3(2):315-25. Disponível em: http://cascavel.ufsm.br/revistas/ojs-2.2.2/index.php/reufsm/article/view/8595

11. Mesquita AC, Chaves ECL, Avelino CCV, Nogueira DA, Panzini RG, Carvalho EC. The use of religious/spiritual coping among patients with cancer undergoing chemotherapy treatment. Rev Latino-Am Enferm [Internet]. 2013 [acesso em: 25 set 2015]; 21(2): 539-45. Disponível em: http://www.scielo.br/pdf/rlae/v21n2/0104-1169-rlae-21-02-0539.pdf

12. Alves RF, Melo MO, Andrade SFO, Fernandes TS, Gonçalves DL, Freire AA.

Qualidade de vida em pacientes oncológicos na assistência

em casas de apoio. Aletheia. 2012; [acesso em: 8 ago 2016]38(39): 39-54. Disponível em: http://pepsic.bvsalud.org/pdf/ aletheia/n38-39/n38-39a04.pdf

13. Hisse CN, Schwartz E, Lima LM, Feijó AM, Santos BP, Viegas AC. Caracterização dos pacientes de quimioterapia e hormonioterapia de uma unidade de oncologia. Rev enferm Cent Oeste Min [Internet]. 2014 [acesso em: 25 set 2015];4(2):1185-93. Disponível em: http://seer.ufsj.edu.br/index.php/recom/article/view/492/753

14 Sehnem S, Veltrini VC. O chimarrão e suas repercussões bucais. Revista Saude e Pesquisa. [internet] 2012; [acesso em: 8 ago 2016] 5(3):447- 53. Disponível em: periodicos.unicesumar.edu.br/index.php/saudpesq/article/download/2062/1800

15. Silva MEDC, Silva LDC, Dantas ALB, Araújo DOR, Duarte IS, Sousa JFM. Assistência de enfermagem ao paciente oncológico no hospital. Rev Enferm UFPI [Internet]. 2013 Dec [acesso em: 25 set 2015];2(5):69-75. Disponível em: http:// www.ojs.ufpi.br/index.php/reufpi/article/view/1359

16. Carmo CBS, Silva RD, Teixeira RC. Perfil epidemiológico de pacientes com câncer de pulmão em hospital público de referência oncológica do estado do Pará. Rev paraense med [Internet]. 2014 [acesso em: 25 set 2015];28(1):55-62. Disponível em: http://files.bvs.br/upload/S/0101-5907/2014/v28n1/a4173.pdf

17. Luz ALA, Luz MHBA, Antunes A, Oliveira GS, Andrade EMLR, Miranda SM. Perfil de pacientes estomizados: revisão integrativa da literatura. Rev Cultura Cuidados [Internet]. 2014 [acesso em: 25 set 2015];18(39):115-23. Disponível em: http://rua.ua.es/dspace/bitstream/10045/40073/1/Cultura_Cuidados_39_13.pdf 